\title{
Using IP networks as a deviceless storage for future portable computers
}

\begin{abstract}
In this paper we propose a generic architecture for a small powerful mobile computer that relies on network and its servers for majority of its activities. Since the network is located in middle of this architecture, we discuss the feasibility and capacity of using the network as a temporary dynamic storage in the form of cache for limited and frequently used data/.control data. However the node delivery and file system design for the proposed network storage is not in the scope of this paper. We show how the routing loop can be utilized to convert the network delay and processing power of the routers to a virtual storage capacity in the network by keeping data in the network in form of floating IP packets. We call this approach Data Storage Technique on IP Networks (DSTN). In satellite and wireless communications this storage can be referred to as deviceless storage. We formulate the potential storage capacity and discuss the parameters that affect the capacity. We validate the technique by comparing the results obtained from the mathematical model with the results obtained from OpNet Modeler simulation tool. Since this paper is a preliminary part of this research, we address the future direction of the research in the last section.
\end{abstract}

Keyword: Deviceless storage; Floating IP; Hop limit; Mobile computer; Routing loop storage; TTL 